How to reference this article Cortelazzo, M. A., Tuzzi, A. (2020). A chi assomiglia Elena Ferrante? Un profilo stilometrico aggiornato. Italica Wratislaviensia, 11(1), 123-141.

DOI: http://dx.doi.org/10.15804/IW.2020.11.1.05

\author{
Michele A. Cortelazzo \\ Università di Padova \\ cortmic@unipd.it \\ ORCID: 0000-0003-2483-7828
}

Arjuna Tuzzi

Università di Padova

arjuna.tuzzi@unipd.it

ORCID: 0000-0003-3795-5567

\title{
A CHI ASSOMIGLIA ELENA FERRANTE? UN PROFILO STILOMETRICO AGGIORNATO
}

\author{
WHO DOES ELENA FERRANTE LOOK LIKE? \\ A REVISED STYLOMETRIC IDENTIKIT
}

\begin{abstract}
Based on a corpus including 150 novels by 40 authors, a stylometric survey was conducted to assess which modern authors were similar to Elena Ferrante, the pen name used for eight novels, including My Brilliant Friend (Tuzzi \& Cortelazzo 2018a and 2018b). The survey proved that Elena Ferrante's writing style is remarkably different from that of the other main contemporary Italian novelists with the notable exception of Domenico Starnone. Follow-up studies (Cortelazzo, Mikros \& Tuzzi 2018 and another under way) show that non-fiction works signed by Elena Ferrante may be attributed to different authors, i.e., Anita Raja, Starnone again, and a collective author including the staff of the E/O publishing house.

This study complements the results obtained by previous research by assessing Elena Ferrante's role in modern Italian fiction following the publication of her latest novel, The Lying Life of Adults. In addition, the analysis of her similarities to Domenico Starnone was enhanced by means of a larger corpus of his novels, thus corroborating the outcome of previous research.
\end{abstract}

Keywords: Elena Ferrante, contemporary Italian literature, authorship attribution, similarity measure, text clustering 


\section{L'ENIGMA ELENA FERRANTE}

Il nome Elena Ferrante è, dichiaratamente, uno pseudonimo dietro il quale si nasconde chi scrive la copiosa produzione letteraria e paraletteraria pubblicata con questo nome: otto romanzi, tra i quali spicca la quadrilogia L'amica geniale (Ferrante, 2011, 2012, 2013, 2014; gli altri romanzi sono Ferrante, 1992, 2002, 2006, 2019a), un racconto per bambini (Ferrante, 2007), una raccolta di articoli apparsi sul "Guardian" e presentati in italiano, probabilmente lingua in cui erano stati scritti gli originali (Ferrante, 2019b), una raccolta di scritti di varia natura (Ferrante, 2016). Si tratta di un corpus ampio e sempre più diversificato, non tutto chiaramente definibile in termini di genere testuale.

La scrittura di Elena Ferrante si presenta, quindi, come un enigma che suscita interesse, perché, come ha osservato Michel Foucault (1971 [1969], pp. 10-11), in un saggio che pure intendeva sottoporre a una critica serrata proprio il concetto di autore tipico della cultura moderna, «l'anonimato letterario non ci è sopportabile, noi lo accettiamo solo come enigma». Proprio per questa esigenza, per molti insopprimibile, l'identificazione dell'effettivo autore di questo corpus letterario ormai cospicuo ha attirato l'attenzione sia di studiosi, sia di giornalisti (oltre che di molti lettori e fan). Ha anche suscitato le reazioni di chi intende non tanto (o non solo) difendere la riservatezza di chi si cela dietro Elena Ferrante, quanto considerare il desiderio di non farsi conoscere nella sua realtà biografica come uno dei punti di forza della sua esperienza letteraria. Rappresenta nella forma più esplicita questa posizione De Rogatis (2018, pp. 20-21), che argomenta così: «Credo che per Ferrante sia una questione fondativa proprio grazie alla scelta (e non nonostante la scelta) di non rivelare la propria reale identità. C'è qualcosa infatti che nessun conto in tasca, nessuna indagine statistica, nessuna invasione della privacy, nessuna ombra di maritale e patriarcale sostegno potranno mai togliere a lei, e a noi lettrici e lettori. In un paese come l'Italia, in cui un costume maschile diffuso nel giornalismo, nell'editoria e nell'università ha delegittimato e delegittima tuttora le nostre scrittrici e la loro visibilità, Elena Ferrante ha scelto di essere una di loro. Portando all'estremo le tante inverosimili illazioni che sono circolate, si potrebbe anche ar- 
rivare a dire per assurdo che questa scrittrice è simultaneamente donna e uomo, travestita/o e transgender, etero e omosessuale, è un singolo essere vivente, una coppia, un trio, un collettivo. Noi non possiamo sapere con certezza assoluta quale sia la verità anagrafica».

L'enigma può essere visto, però, da una prospettiva diversa, che non miri all'identità anagrafica, ma all'identità letteraria. Chi si occupa di attribuzione d'autore (Autorship attribution) con strumenti di analisi quantitativa confronta testi. L'oggetto di analisi, quindi, non è la biografia dell'autore, ma la configurazione linguistica dei testi; quello che si può determinare non sono le caratteristiche personali dell'autore, ma le similarità della sua scrittura rispetto a quella di altri autori, quale si ricava non da una sistematica e puntuale lettura delle opere che si ritiene di dover confrontare, ma da una visione «dall'alto», che può essere rappresentata, in forma sintetica, da tabelle e grafici. Le similarità che così emergono indicano l'esistenza di affinità stilistiche e permettono di inquadrare meglio lo stile delle opere pubblicate sotto pseudonimo nel quadro delle opere letterarie coeve; similarità particolarmente forti possono essere segnale di un'unità stilistica tale da rendere probabile anche un'unità autoriale.

Il tentativo di individuare gli autori che risultano stilometricamente vicini a un autore anonimo possono essere interessanti, anche dal punto di vista critico, indipendentemente dal riconoscimento dell'identità reale dell'autore: la rilevante distanza di un autore anonimo dalla fisionomia dei principali autori contemporanei può indicare l'estraneità dell'autore in esame all'ambiente letterario conosciuto (può far supporre, cioè, che si tratti di un autore che ha prodotto solo gli scritti raggruppati sotto lo pseudonimo); la sovrapponibilità delle opere dell'autore anonimo a quelle di altri autori può parlare a favore dell'esistenza di un filone stilistico unitario e nitidamente riconoscibile, di cui fanno parte gli autori coinvolti, anche senza necessariamente ipotizzare un'identità autoriale (con conseguenze rilevanti sul piano critico se le caratteristiche degli autori coinvolti sono diverse, per esempio in termini di genere, di età o di provenienza regionale, da quelle attribuite all'autore anonimo); la similarità stilometrica dell'autore investigato con gli scritti di autori che hanno un'esperienza di scrittura diversa da quella 
narrativa (per esempio saggistica, giornalistica o traduttiva) può portare a importanti riflessioni sulla tenuta delle differenziazioni dei generi della scrittura.

I tentativi di identificare Elena Ferrante sono stati oggetto, fino al 2017, solo di dibattiti giornalistici o sul web. Alcuni hanno cercato di individuare l'identità anagrafica dell'autore o dell'autrice che si firma Elena Ferrante, operando su dati extra-testuali (per esempio cercando di riconoscere chi sia il beneficiario dei diritti d'autore: Gatti, 2016) oppure fondandosi su evidenze biografiche deducibili dall'ambientazione topografica e cronologica dei romanzi (Santagata, 2016). Altri hanno cercato di individuare affinità tematiche e lessicali tra le opere di Elena Ferrante e quelle di altri autori contemporanei (si sono indirizzati in questa direzione principalmente Galella, 2005 e 2006, anche sulla scorta di rilievi quantitativi del fisico Vittorio Loreto, e Gatto, 2006 e 2016). Ulteriori ipotesi provengono da intuizioni di critici, giornalisti, letterati. In tutti i tentativi si è partiti da un presupposto non dimostrabile, ma giustificato dalla maturità di scrittura attestata fin dall'opera prima, precisamente dal presupposto che chi si nasconde dietro lo pseudonimo Elena Ferrante abbia scritto altre opere, narrative o di altro genere.

Per questo, chi si è occupato della attribuzione con mezzi quantitativi delle opere di Elena Ferrante ha adottato una logica di authorship identification, che presume di poter determinare un insieme chiuso di possibili candidati con i quali confrontare le opere da attribuire, nell'ipotesi che l'autore delle opere anonime sia uno degli autori individuati. Questa direzione si è rafforzata nel corso degli anni, via via che gli studi stilistici, tematici, biografici o le indagini extratestuali hanno circoscritto il novero degli intellettuali sospettati di nascondersi dietro il nome di Elena Ferrante.

Anche noi ci siamo occupati dell'attribuzione delle opere di Elena Ferrante, nella prospettiva di definirne l'identità letteraria in termini di similarità stilistica determinabile con strumenti quantitativi. Abbiamo costituito un corpus ad hoc (denominato "PIC, Padua Italian Corpus" da Savoy, 2018a), adeguato allo scopo di inserire Elena Ferrante nel contesto dei principali autori contemporanei italiani e di avere elementi per affrontare due temi che sono stati oggetto di riflessioni in ambito critico: 
la napoletanità di Elena Ferrante e la significatività delle sue opere come esempi di scrittura di genere.

Il corpus comprende 150 romanzi scritti originariamente in italiano, usciti, con tre sole eccezioni, tra il 1987 e il 2016 (compresi i primi sette romanzi di Elena Ferrante), opera di 40 autori diversi, scelti in modo che fossero adeguatamente rappresentati autori o autrici di area campana (cioè l'area geografica da cui dichiara di provenire Elena Ferrante), autrici di genere femminile, autori o autrici sospettati di essere Elena Ferrante, autori o autrici di bestseller (considerati tali perché hanno avuto un successo di vendite o hanno ottenuto prestigiosi premi letterari), autori o autrici di romanzi che risultano di particolare interesse secondo il giudizio raccolto tra esperti di letteratura contemporanea.

I 150 romanzi sono di questi autori: oltre a Elena Ferrante, Eraldo Affinati, Niccolò Ammaniti, Andrea Bajani, Marco Balzano, Alessandro Baricco, Stefano Benni, Enrico Brizzi, Gianrico Carofiglio, Mauro Covacich, Erri De Luca, Diego De Silva, Giorgio Faletti, Marcello Fois, Paolo Giordano, Nicola Lagioia, Dacia Maraini, Margareth Mazzantini, Melania Mazzucco, Rossella Milone, Giuseppe Montesano, Marta Morazzoni, Michela Murgia, Edoardo Nesi, Paolo Nori, Valeria Parrella, Francesco Piccolo, Tommaso Pincio, Michele Prisco, Christian Raimo, Fabrizia Ramondino, Ermanno Rea, Tiziano Scarpa, Clara Sereni, Domenico Starnone, Susanna Tamaro, Chiara Valerio, Giorgio Vasta, Sandro Veronesi e Simona Vinci. Tra questi, se si esclude Elena Ferrante, 10 sono autori campani e 12 sono donne.

Il corpus così raccolto si presenta come un corpus di grandi dimensioni (consiste in 9.837.851 occorrenze, cioè tokens) ed è formato, con un'unica eccezione, da romanzi lunghi almeno 10.000 parole; esprime complessivamente un vocabolario di 159.149 forme diverse (types).

\section{LA COLLOCAZIONE DI ELENA FERRANTE NEL PANORAMA LETTERARIO ITALIANO CONTEMPORANEO}

Basandoci sul corpus così costituito, abbiamo condotto una ricerca, i cui risultati sono stati pubblicati in Tuzzi \& Cortelazzo (2018a), al quale rinviamo per la discussione dettagliata di metodi e conclusioni e per la 
rappresentazione grafica dei dati raccolti. Qui riassumiamo le conclusioni più importanti.

Il modo in cui è stato costituito il corpus ci ha permesso di affrontare tre aspetti: il primo, più generale, riguarda la comparazione delle opere di Elena Ferrante con quelle dei più significativi autori italiani contemporanei, per capire se il successo internazionale delle opere dell'autrice (enormemente superiore al successo che negli ultimi decenni ha arriso alle opere letterarie italiane) possa avere un riscontro nello stile autoriale; un secondo, orientato alla ricerca di una differenziazione regionale della letteratura italiana contemporanea, ha cercato di verificare se le peculiarità individuate nella scrittura di Elena Ferrante siano legate all'ambientazione napoletana delle vicende narrate, all'origine napoletana dichiarata da Elena Ferrante, o comunque a una sorta di koinè letteraria regionale; il terzo, legato a una prospettiva di genere, ha l'obiettivo di valutare quanto la scrittura di Elena Ferrante sia omogenea alla scrittura delle scrittrici italiane degli ultimi anni.

Sono state usate tecniche che, basandosi su un'analisi quantitativa del lessico delle opere confrontate, permettesse di leggere «da distante» (per rifarci alla nota espressione di Moretti, 2013) l'insieme delle opere e di vedere in un colpo d'occhio la collocazione reciproca di tutti gli autori e di tutte le opere oggetto del nostro studio. Le tecniche usate sono state due: un'analisi, esplorativa, condotta attraverso l'analisi delle corrispondenze (Greenacre, 1984) e un'analisi effettuata attraverso la misura della distanza intertestuale di Labbé (Labbé \& Labbé, 2001, 2007) che, pur presentando alcuni limiti (Viprey \& Ledoux, 2006), si è rivelata efficace negli esperimenti condotti su testi di certa attribuzione, soprattutto nella versione iterativa da noi proposta (Cortelazzo, Nadalutti \& Tuzzi, 2013; Tuzzi, 2010).

L'analisi delle corrispondenze applicata all'intero corpus ha fatto emergere tre autori che appaiono i più originali; tra questi Elena Ferrante (gli altri due sono Paolo Nori e Giorgio Faletti) mostra la più marcata individualità tematica e di scrittura, finendo isolata e rappresentata a notevole distanza anche dagli scrittori che evidenziano qualche affinità, come i meridionali (in gran parte napoletani) Prisco, Rea, Starnone, De Luca, Montesano, Carofiglio, il milanese, ma di famiglia 
meridionale, Balzano e, fuori da questo quadro, la romana Clara Sereni. L'autore che le risulta più vicino, e quindi più simile in termini di profilo lessicale, è Domenico Starnone. In Tuzzi \& Cortelazzo (2018a, p. 689, figura 1) si trova la rappresentazione visiva di questi risultati, presentati sia dettagliatamente per romanzo, sia complessivamente per autore.

Se ci si focalizza sul subcorpus costituito dalle tredici autrici, l'analisi delle corrispondenze fa emergere un'immagine ancora più chiara della posizione di Elena Ferrante: 1'autrice si trova in pieno isolamento, in una posizione di evidente e assoluta autonomia rispetto a quella di tutte le altre scrittrici, con una leggera somiglianza solo con Rossella Milone, autrice più giovane delle altre, che può aver individuato in Elena Ferrante un modello di riferimento. La configurazione dei singoli romanzi mostra qualche moderata affinità solo con uno dei romanzi di Michela Murgia e uno di Clara Sereni (la rappresentazione grafica è in Tuzzi \& Cortelazzo, 2018a, p. 690, figura 2: anche in questo caso i risultati sono presentati sia per i singoli romanzi, sia complessivamente per autore).

Infine, anche l'analisi del subcorpus costituito da Elena Ferrante e dagli altri dieci autori e autrici di area campana conferma la peculiarità della posizione di Elena Ferrante, la cui produzione appare coesa (con una parziale differenziazione solo del primo romanzo, che si colloca in uno spazio occupato anche da romanzi di De Luca, Milone, Da Silva). Si riconosce, poi, una vicinanza ai romanzi di Domenico Starnone pubblicati successivamente al 1993. Emerge anche una particolarità della posizione di Starnone, le cui opere si raccolgono in due nuclei ben distinti: da una parte i romanzi del primo periodo (1987-1991), situati in una posizione autonoma e appartata, dall'altra quelli dal 1993 in poi, vicini ai romanzi di Elena Ferrante. La rappresentazione grafica, anche in questo caso presentata sia per singoli romanzi, sia complessivamente per autore si trova nella figura 3 riportata in Tuzzi \& Cortelazzo (2018a, p. 690).

A sua volta, il dendrogramma che presenta graficamente i risultati delle misure della distanza intertestuale di Labbé conferma i risultati dell'analisi delle corrispondenze: identifica un cluster che include tutti 
i romanzi scritti da Ferrante e buona parte dei romanzi di Starnone, con l'esclusione dei primi tre che vanno a costituire un piccolo cluster autonomo.

I risultati della misura della distanza intertestuale risultano ancor meglio leggibili se vengono presentati sotto forma di ranking, individuando, per ogni romanzo, quali sono i romanzi del corpus che si situano più vicini al romanzo preso in esame. Per quel che riguarda Elena Ferrante, si può osservare che per tutte le sue opere, tranne la prima, le prime posizioni sono occupate da un'altra opera della stessa autrice. In particolare, tutti i romanzi della quadrilogia risultano molto simili tra di loro, come peraltro ci si aspetta da una storia in quattro puntate; nelle posizioni immediatamente successive mostrano la massima somiglianza con i romanzi più recenti di Domenico Starnone. Una parziale eccezione rispetto a questo quadro generale è costituito dal primo romanzo di Ferrante, $L$ 'amore molesto, che presenta un profilo particolare, in quanto il romanzo che appare più simile è un romanzo di Domenico Starnone (Eccesso di zelo, uscito nell'anno successivo, il 1993). Lo stesso romanzo appare in seconda posizione per i successivi due romanzi di Elena Ferrante, I giorni dell'abbandono e La figlia oscura.

Vista la configurazione dei ranking relativi alle opere di Elena Ferrante è parso importante verificare anche quelli relativi alle opere di Domenico Starnone. I romanzi di quest'ultimo, a partire da Eccesso di zelo, presentano in prima posizione almeno un'opera di Elena Ferrante, con il risultano paradossale che le opere di Starnone risultano più simili alle opere di Elena Ferrante che a quelle dell'autore stesso. Solo nelle successive posizioni sono presenti altri romanzi di Starnone e poi, spesso, compaiono opere di Francesco Piccolo, altro autore napoletano. I ranking delle prime opere di Starnone presentano, invece, una configurazione del tutta diversa: la prima opera (Ex cattedra) non mostra somiglianze con i romanzi di Elena Ferrante, mentre nei ranking del secondo e del terzo romanzo le opere di Elena Ferrante iniziano ad apparire solo a partire dalla settima o dalla ottava posizione.

Questo quadro diventa ancora più nitido se misuriamo la distanza intertestuale basandoci solo sulle parole grammaticali (articoli, preposizioni, congiunzioni). La similarità tra i testi di Ferrante e quelli di Star- 
none risulta, quindi, essere indipendente da somiglianze di ambientazione o di sviluppo narrativo.

Il corpus utilizzato per la ricerca di Tuzzi \& Cortelazzo (2018a) è stato oggetto anche di un esercizio di attribuzione con metodi quantitativi, di natura diversa, realizzato, nel corso di un workshop, all'interno della "IQLA-GIAT Summer School in Quantitative Analysis of Textual Data" (Università di Padova, 4-8 settembre 2017), da importanti studiosi di Authorship attribution e di analisi statistica dei dati testuali La vicinanza tra le opere di Elena Ferrante e quelle di Domenico Starnone è stata confermata anche dalle ricerche presentate in tale occasione (Eder, 2018; Juola, 2018; Lalli, Tria, Loreto, 2018; Mikros, 2018; Ratinaud, 2018; Rybicki, 2018; Savoy, 2018) e in studi successivi (Savoy, 2018b; Cortelazzo, Mikros \& Tuzzi, in preparazione).

\section{VERIFICHE SU TESTI NON LETTERARI}

Il confronto della scrittura di Elena Ferrante con quella di altri autori non può essere considerato esaurito dallo studio del corpus di romanzi contemporanei, per quanto i risultati siano di inusuale nettezza. Tra quanti sono stati segnalati come possibili autori effettivi delle opere di Elena Ferrante vi sono alcune autrici che non hanno mai scritto romanzi, anche se hanno all'attivo altre importanti esperienza di scrittura. Ci riferiamo specificamente a Marcella Marmo (storica, professoressa, ora in pensione, all'Università "Federico II" di Napoli) e Anita Raja (traduttrice dal tedesco, per conto proprio della casa editrice E/O, moglie di Domenico Starnone).

È stato quindi necessario costituire un secondo corpus, che includesse gli scritti non narrativi di Elena Ferrante (compresi nel volume La Frantumaglia, nel quale sono state raccolte lettere, risposte a interviste scritte, altri materiali: Ferrante, 2016). Questi scritti vanno raffrontati con quelli, di genere analogo, di altri autori: innanzi tutto le due autrici appena ricordate e Domenico Starnone, che dagli studi sulle opere letterarie appariva l'autore più prossimo a Elena Ferrante; poi altri autori o autrici il cui nome è stato, in varie occasioni, avanzato, anche provocatoriamente, nelle discussioni sull'identità di Elena Ferrante (Gof- 
fredo Fofi, Mario Martone, Valeria Parrella, Francesco Piccolo, Laura Buffoni) e autori che nell'analisi dei romanzi avevano mostrato qualche tratto in comune con Elena Ferrante (Gianrico Carofiglio, Clara Sereni). Inoltre è stato creato un autore fittizio, che rappresenta collettivamente la casa editrice $\mathrm{E} / \mathrm{O}$ (sono stati attribuiti a questo autore scritti dei proprietari della casa editrice, Sandro Ferri e Sandra Ozzola e pagine editoriali tratte dal sito web della casa editrice). Il corpus presenta un limite difficilmente superabile, in quanto contiene diversi tipi di testo (saggi, interviste, articoli di giornali, lettere, prefazioni o postfazioni, trascrizioni di conferenze declamate a partire da un testo scritto): per quanto i testi utilizzati abbiano dei tratti in comune (affrontano temi legati alla letteratura, ma non si presentano come testi letterari), la loro diversità tipologica può influire sui risultati, in quanto le somiglianze tra $i$ testi analizzati possono essere fatti risalire alla affinità tra gli autori, ma anche alla similarità del tipo di testo, senza che gli effetti di questi due parametri si possano facilmente districare.

Complessivamente il corpus è costituito da 113 testi, per un totale di 143.120 occorrenze e 18.757 parole diverse. La lunghezza dei testi va da un minimo di 227 parole e un massimo di 8.977. Il corpus è ben bilanciato dal punto di vista del genere e sufficientemente equilibrato dal punto di vista dell'età e della provenienza geografica.

Questo corpus è stato utilizzato in due lavori presentati in collaborazione con George Mikros. In Cortelazzo, Mikros \& Tuzzi (2018) abbiamo utilizzato metodi standard di classificazione dell'autore e tecniche di profilazione. Dopo una fase preliminare abbiamo ritenuto utile ridurre i candidati, considerando quelli che sono apparsi più significativi al primo esame: Starnone, Raja, Martone e l'autore collettivo E/O. I risultati della profilazione suggeriscono il coinvolgimento di almeno un uomo e una donna, disegnano il ritratto di un autore (singolo o collettivo) originario di Napoli, con un'età superiore ai 60 anni.

In Cortelazzo et al. (in preparazione) abbiamo applicato il General Impostors' method (Kestemont, Stover, Koppel, Karsdorp \& Daelemans, 2016a, 2016b; Koppel \& Winter, 2014; Koppel, Schler \& Argamon, 2011). Anche con questo metodo, i testi di Elena Ferrante tratti dalla Frantumaglia appaiono essere stati scritti da più mani. Come pos- 
sibili autori affiorano Starnone, Raja, Martone, Parrella e lo staff della casa editrice $\mathrm{E} / \mathrm{O}$.

A differenza di quello che è accaduto con il corpus letterario, dalle analisi del corpus non letterario non si ricavano risultati decisivi. Si possono estrarre comunque dei dati interessanti. Innanzi tutto, si possono escludere con sufficiente certezza Buffoni, Carofiglio, Ferri, Fofi, Marmo, Piccolo, che in nessuna delle indagini condotte entrano nel novero dei candidati. Naturalmente, l'informazione più significativa, rispetto alle attese suscitate dalle discussioni sviluppatesi a proposito dell'identità di Elena Ferrante, è la chiara estraneità di Marcella Marmo.

Inoltre, un esito assodato è che i testi saggistici di Elena Ferrante non presentano un profilo stilometrico omogeneo e possono, anzi, probabilmente, devono essere attribuiti a una pluralità di autori. Questa conclusione va interpretata in due modi: da una parte si deve ipotizzare che alcuni testi siano stati scritti da un autore, e altri da un altro, dall'altra che alcuni testi appaiono il frutto di un processo di scrittura cooperativa o hanno la fisionomia di un collage di contributi di diversi autori. Risulta importante che tra i potenziali autori presi in esame, tutte le indagini abbiano incluso sia Domenico Starnone, sia Anita Raja, sia la casa editrice $\mathrm{E} / \mathrm{O}$. È interessante anche che in entrambi i lavori di cui abbiamo dato un resoconto compaia il nome di Mario Martone: fa parte del gruppo impegnato a dare le risposte a chi intervista Elena Ferrante o la sua presenza è un effetto indiretto delle interazioni epistolari intercorse tra Martone e Ferrante, nel periodo in cui veniva messa a punto la sceneggiatura della trasposizione cinematografica del romanzo L'amore molesto? Resta da chiarire anche la presenza tra i possibili autori di Valeria Parrella, il cui nome emerge solo dall'applicazione del General Impostors'method.

\section{UN AGGIORNAMENTO: FERRANTE E STARNONE (ANCHE DOPO L'AMICA GENIALE)}

Le ricerche che abbiamo finora condotto su Elena Ferrante ci portano, quindi, ad alcune conclusioni certe e ad alcuni risultati che richiedono approfondimenti, con l'obiettivo di avere una visione più chiara dei rap- 
porti tra gli scritti firmati da Elena Ferrante e altri prodotti di scrittura di questi anni.

Sul piano della scrittura letteraria sono due i punti che escono con chiarezza dalle indagini: innanzi tutto la particolarità, ma anche la forte coerenza, della scrittura di Elena Ferrante, che si configura come un unicum nel panorama letterario contemporaneo; poi l'affinità che comunque, pur da questa posizione particolare, la scrittrice presenta con una parte considerevole dell'opera narrativa di Domenico Starnone, ma non con tutta.

Sul piano della scrittura non letteraria emerge, invece, il sospetto di una non completa identità autoriale: i contatti che Elena Ferrante tiene, attraverso l'editore, con il mondo esterno, quando concede interviste o discute con chi si immerge professionalmente nel suo mondo letterario (per esempio per trasformarlo in un prodotto filmico), non mostrano, a un'analisi quantitativa, la stessa monoliticità e coerenza stilistica delle opere letterarie. L'ipotesi è che negli scritti raccolti nella Frantumaglia affiorino le tracce di mani diverse (e anche qualche indizio di scrittura cooperativa). Certamente, in questa caratterizzazione poliforme degli scritti non letterari firmati da Elena Ferrante, è plausibile il contributo di Anita Raja e di Domenico Starnone. Ma, a differenza di quanto accade per la scrittura letteraria, i risultati delle indagini quantitative suscitano dubbi e sospetti, ma non portano (ancora?) a certezze sul profilo lessicale degli scritti della Frantumaglia, sicuramente anche a causa della limitatezza del corpus analizzabile.

Rispetto al momento nel quale sono state condotte le ricerche alle quali ci siamo riferiti nei paragrafi precedenti, il corpus di Elena Ferrante si è arricchito di due opere, uscite entrambe nel 2019: il romanzo La vita bugiarda degli adulti (Ferrante, 2019a) e la raccolta L'invenzione occasionale (Ferrante, 2019b), costituita dai testi pubblicati sul Guardian nel corso del 2018 (qui nella versione in inglese di Ann Goldstein). A sua volta, Domenico Starnone ha pubblicato, sempre nel 2019, un nuovo romanzo, Confidenze. È difficile decidere una netta attribuzione di genere letterario a Ferrante (2019b): se da una parte i brevi interventi che confluiscono nel volume rappresentano delle riflessioni dell'autrice sulla sua esperienza di vita, e in particolare sul suo avvio alla scrittura, 
in modo da risultare affine alla Frantumaglia, molti accenti della ricostruzione di questo processo, e di altri momenti della vita giovanile, ricalcano la rappresentazione della crescita di Elena Greco, come emerge nella Tetralogia (come ha mostrato Gargano, 2019) e, di conseguenza, il volume potrebbe affiancarsi alle opere narrative. Per queste ragioni, il volume non entra pienamente a far parte di nessuno dei due insiemi e non è stato quindi inserito nel corpus.

In questa situazione, è risultato opportuno innanzi tutto aggiornare i risultati delle nostre ricerche alla luce dell'uscita del nuovo romanzo di Elena Ferrante. L'inserimento del nuovo romanzo di Elena Ferrante nel corpus dei 150 romanzi già analizzati (che con l'inserzione di La vita bugiarda degli adulti diventano 151) non ha provocato nessuno sconvolgimento nella mappa che proviene dall'analisi delle corrispondenze: la posizione di Elena Ferrante rimane isolata a conferma della sua particolarità nel panorama della narrativa italiana. Il romanzo del 2019 si pone sulla scia dell'Amica geniale.

L'occasione, però, si è prestata per approfondire il rapporto tra la scrittura di Elena Ferrante e quella di Domenico Starnone: i risultati delle indagini già pubblicate, infatti, non solo ci fanno riconoscere le affinità tra i due autori, ma ci sollecitano a riconoscere un'evoluzione nell'esperienza letteraria di Starnone, che propone una scansione cronologica che viene a coincidere con date fondamentali della produzione letteraria di Elena Ferrante.

Abbiamo, così, sottoposto alla misura della distanza intertestuale un nuovo corpus, che, per quel che riguarda Elena Ferrante, include anche il romanzo del 2019 e, per quel che riguarda Domenico Starnone, è stato ampliato, aggiungendo altri quattro romanzi (Labilità del 2005, Spaventi del 2009, Fare scene del 2010, Confidenze del 2019) ai dieci che facevano già parte del PIC.

Gli esiti della misura della distanza intertestuale sono rappresentati nella Fig. 1, costituita dal dendrogramma che rappresenta i cluster ottenuti con un algoritmo di classificazione gerarchico agglomerativo con metodo del legame completo basato sulle distanze intertestuali di Labbé, calcolate per tutte le coppie di romanzi con metodo iterativo (200 replicazioni, porzioni di testo da 5.000 parole). Si tratta di una riproposizio- 
ne, in base al corpus ridotto di 22 opere appena descritto, dell'analisi presentata nella figura 4 di Tuzzi \& Cortelazzo (2018, p. 692).

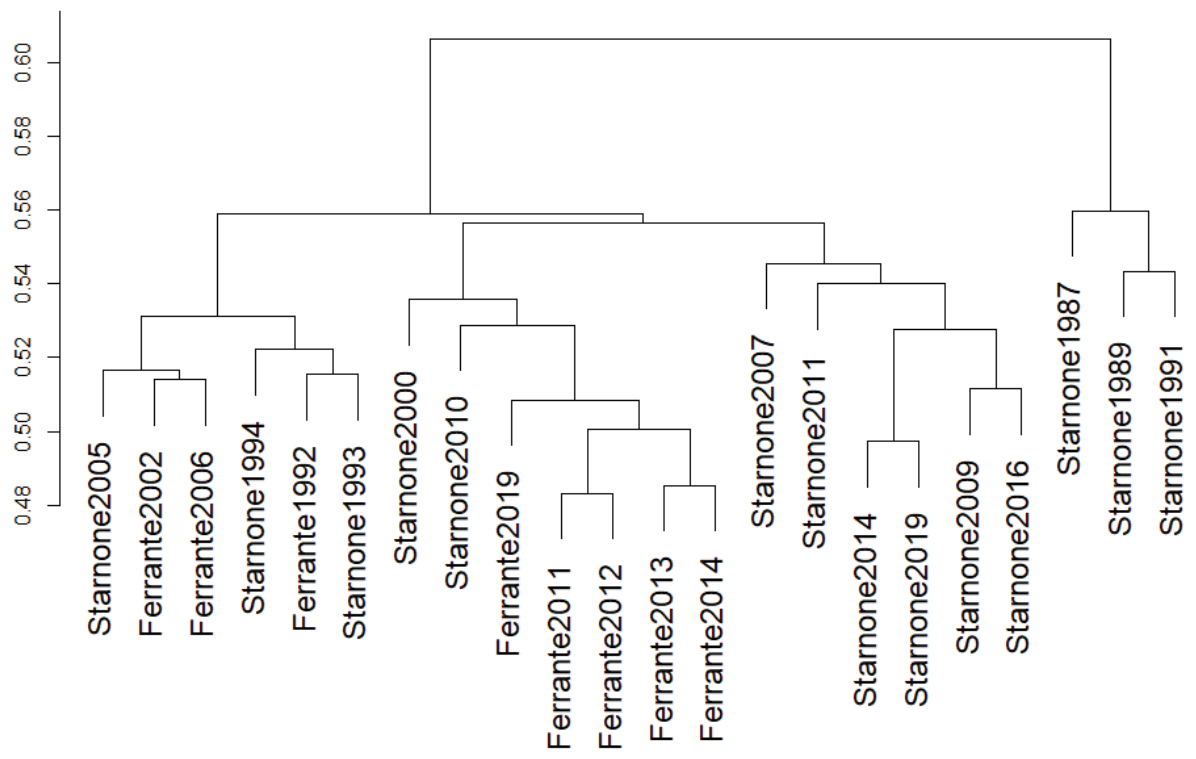

Fig. 1. Dendrogramma. Classificazione dei 22 romanzi scritti da Elena Ferrante e Domenico Starnone

Come si vede, i romanzi esaminati si aggregano secondo lo schema già emerso dalla ricerca del 2018 (Tuzzi \& Cortelazzo, 2018): il primo nodo separa le opere iniziali di Domenico Starnone (dal 1987 al 1991) da tutte le altre. Le opere successive si mescolano con quelle di Elena Ferrante, in quanto dipendono dallo stesso nodo, con una ripartizione in tre blocchi: nel primo sono comprese le prime opere di Elena Ferrante e i romanzi pubblicati da Starnone tra il 1993 e il 2005 (con l'eccezione di Via Gemito del 2002); del secondo fa parte la quadrilogia dell'Amica geniale, alla quale si aggancia pienamente il romanzo più recente, e due romanzi di Starnone, Via Gemito e Fare scene, tematicamente affine, in una sua parte, a Via Gemito; del terzo, che raggruppa solo opere di Domenico Starnone, troviamo i suoi romanzi più recenti. 
L'evoluzione dell'esperienza letteraria di Starnone sembra, quindi, correre in qualche modo parallela a quella di Elena Ferrante. Negli anni in cui inizia a scrivere Elena Ferrante, il profilo lessicale di Starnone, come viene rappresentato dalla distanza intertestuale, subisce un deciso rinnovamento, così come le narrazioni ambientate in via Gemito mostrano delle affinità più strette con la tetralogia e il romanzo più recente di Elena Ferrante. Le opere più recenti indicano, invece, una sorta di riacquisizione di autonomia letteraria da parte di Domenico Starnone.

Il disegno che ne emerge è compatibile con i risultati della ricerca di Eder (2018) che, partendo dall'ipotesi di una sovrapponibilità di Elena Ferrante e Domenico Starnone, ha mostrato un percorso analogo a quello da noi evidenziato: Starnone parte da uno stile pienamente autonomo, per poi giungere gradualmente a intersecarsi con gli esiti di Elena Ferrante, per recuperare, negli ultimi anni, una più marcata fisionomia stilistica individuale.

Questa ricostruzione dei rapporti tra la scrittura di Domenico Starnone e quella di Elena Ferrante non implica necessariamente un'identificazione di quest'ultima con il primo e potrebbe essere spiegata in termini di un primo avvicinamento e poi un allontanamento di due scrittori, entrambi di successo (anche all'estero), che trattano temi analoghi. Ma le affinità emergono con nettezza. Nella ricostruzione di questo processo di avvicinamento e allontanamento incombe, però, un limite: nella successione cronologica noi possiamo basarci esclusivamente sulla data di pubblicazione dei romanzi. Ma non c'è nulla che ci garantisca, per esempio, che La vita bugiarda degli adulti sia stata davvero composta dopo la conclusione della tetralogia dell'Amica geniale o che la stesura di Fare scene sia davvero così distante dal momento della scrittura di Via Gemito (Starnone ha dichiarato che «questi e altri appunti - tutto quello che c'è in queste pagine fino alla fine - sono stati buttati giù anni fa e dovevano servirmi per un paio di libri che avevo in mente. I libri poi li ho scritti, ma non sono riuscito a utilizzare tutto il materiale preparatorio»: Puglisi, 2010). 


\section{BIBLIOGRAFIA}

Cortelazzo, M.A., Mikros, G.K., \& Tuzzi, A. (to appear). Applying General Impostors' Method to the Ferrante Case, conference paper "Digital Stylistics in Romance Studies and Beyond" (Würzburg, 27 February-2 March 2019).

Cortelazzo, M.A., Mikros, G.K., \& Tuzzi, A. (2018). Profiling Elena Ferrante: A Look Beyond Novels. In D. Fioredistella Iezzi, L. Celardo, \& M. Misuraca (Eds.), JADT 2018: Proceedings of the 14th International Conference on Statistical Analysis of Textual Data (pp. 165-173). Roma: UniversItalia.

Cortelazzo, M.A., Nadalutti, P. \& Tuzzi, A. (2013). Improving Labbé's Intertextual Distance: Testing a Revised version on a Large Corpus of Italian Literature. Journal of Quantitative Linguistics, 20(2), 125-152. doi: https://doi.org/10.1080/09296174.2013.773138.

De Rogatis, T. (2018). Elena Ferrante. Parole chiave. Roma: E/O.

Eder, M. (2018). Elena Ferrante: A Virtual Author. In A. Tuzzi, \& M.A. Cortelazzo (2018), Drawing Elena Ferrante's Profile. Workshop Proceedings, Padova, 7 September 2017 (pp. 31-45). Padova: Padova University Press.

Ferrante, E. (1992). L'amore molesto. Roma: E/O.

Ferrante, E. (2002). I giorni dell'abbandono. Roma: E/O.

Ferrante, E. (2006). La figlia oscura. Roma: E/O.

Ferrante, E. (2007). La spiaggia di notte. Roma: E/O.

Ferrante, E. (2011). L'amica geniale. Infanzia, adolescenza. Roma: E/O.

Ferrante, E. (2012). Storia del nuovo cognome. L'amica geniale volume secondo. Roma: E/O.

Ferrante, E. (2013). Storia di chi fugge e di chi resta. L'amica geniale volume terzo. Roma: E/O.

Ferrante, E. (2014). Storia della bambina perduta. L'amica geniale volume quarto. Roma: E/O.

Ferrante, E. (2016). La Frantumaglia. Roma: E/O.

Ferrante, E. (2019a). La vita bugiarda degli adulti. Roma: E/O.

Ferrante, E. (2019b). L'invenzione occasionale. Roma: E/O.

Foucault, M. (1969). Qu'est-ce qu'un auteur? Bulletin de la Société française de philosophie, 3 (luglio-settembre), 73-104 (trad. it. Che cos'è un autore? in Scritti letterari, Milano, Feltrinelli, 1971, pp. 1-21). 
Galella, L. (2005, 16 January). Ferrante-Starnone. Un amore molesto in via Gemito. La Stampa, 27.

Galella, L. (2006, November 23). Ferrante è Starnone. Parola di computer. L'Unità, 26.

Gargano, C. (2019). La giusta distanza. In D. La Monaca, \& D. Perrone (Eds.), Incontro con Elena Ferrante (pp. 101-111). Palermo: Palermo University Press.

Gatti, C. (2016, October 2). Elena Ferrante, le «tracce» dell'autrice identificata. Il Sole 24 Ore - Domenica, 1-2.

Gatto, S. (2006, October 28). Starnone-Ferrante: quando il senso di colpa genera doppi. Lo Specchio di carta. Osservatorio sul romanzo italiano contemporaneo, [Blog post]. Retrieved from https://lospecchiodicarta.it/2006/10/28/starnone-ferrante-quando-il-senso-di-colpa-generadoppi.

Gatto, S. (2016, October 22). Una biografia, due autofiction. Ferrante-Starnone: cancellare le tracce, [Blog post]. Retrieved from https://lospecchiodicarta.it/2016/10/22/una-biografia-due-autofiction-ferrante-starnone-cancellare-le-tracce.

Greenacre, M.J. (1984). Theory and Application of Correspondence Analysis. London: Academic Press.

Juola, P. (2018). Thesaurus-Based Semantic Similarity Judgments: A New Approach to Authorial Similarity? In A. Tuzzi, \& M.A. Cortelazzo (2018), Drawing Elena Ferrante's Profile. Workshop Proceedings, Padova, 7 September 2017 (pp. 47-59). Padova: Padova University Press.

Kestemont, M., Stover, J. Koppel, M., Karsdorp, F., \& Daelemans, W. (2016a). Authorship Verification with the Ruzicka Metric. In Digital Humanities 2016: Conference Abstracts (pp. 246-249). Krakow: Jagiellonian University \& Pedagogical University.

Kestemont, M., Stover, J., Koppel, M., Karsdorp, F., \& Daelemans, W. (2016b). Authenticating the writings of Julius Caesar. Expert Systems with Applications, 63, 86-96. doi: https://doi.org/10.1016/j.eswa.2016.06.029.

Koppel, M., \& Winter, Y. (2014). Determining if two documents are written by the same author. Journal of the Association for Information Science and Technology, 65(1), 178-187. doi: https://doi.org/10.1002/asi.22954.

Koppel, M., Schler, J., \& Argamon, S. (2011). Authorship attribution in the wild. Language Resources and Evaluation, 45(1), 83-94. doi: 10.1007/ s10579-009-9111-2. 
Labbé, C., \& Labbé, D. (2001). Inter-Textual Distance and Authorship Attribution Corneille and Molière. Journal of Quantitative Linguistics, 8(3), 213-231. doi: https://doi.org/10.1076/jqul.8.3.213.4100.

Labbé, D. (2007). Experiments on authorship attribution by intertextual distance in English. Journal of Quantitative Linguistics, 14(1), 33-80. doi: https://doi.org/10.1080/09296170600850601.

Lalli, M., Tria, F., \& Loreto, V. (2018). Data-Compression Approach to Authorship Attribution. In Tuzzi \& Cortelazzo (2018), p. 61-83

Mikros, G. (2018). Blended Authorship Attribution: Unmasking Elena Ferrante Combining Different Author Profiling Methods. In A. Tuzzi, \& M.A. Cortelazzo (2018). Drawing Elena Ferrante's Profile. Workshop Proceedings, Padova, 7 September 2017 (pp. 85-95). Padova: Padova University Press.

Moretti, F. (2013). Distant Reading. London: Verso.

Puglisi, A. (2010, November 7). "Fare scene" di Domenico Starnone [Blog post]. Retrieved from: http://www.sulromanzo.it/blog/fare-scene-di-domenico-starnone.

Ratinaud, P. (2018). The Brilliant Friend(s) of Elena Ferrante: A Lexicometrical Comparison between Elena Ferrante's Books and 39 Contemporary Italian Writers. In A. Tuzzi, \& M.A. Cortelazzo (2018), Drawing Elena Ferrante's Profile. Workshop Proceedings, Padova, 7 September 2017 (pp. 97-110). Padova: Padova University Press.

Rybicki, J. (2018). Partners in Life, Partners in Crime? In A. Tuzzi, \& M.A. Cortelazzo (2018), Drawing Elena Ferrante's Profile. Workshop Proceedings, Padova, 7 September 2017 (pp. 111-122). Padova: Padova University Press.

Santagata, M. (2016, March 13). Elena Ferrante è ... La lettura - Corriere della Sera, 2 \& 5.

Savoy, J. (2018a). Elena Ferrante Unmasked. In A. Tuzzi, \& M.A. Cortelazzo (2018), Drawing Elena Ferrante's Profile. Workshop Proceedings, Padova, 7 September 2017 (pp. 129-139). Padova: Padova University Press.

Savoy, J. (2018b). Is Starnone really the author behind Ferrante? Digital Scholarship in the Humanities, 33(4), 902-918. doi: https://doi.org/10.1093/ llc/fqy016.

Tuzzi, A. (2010). What to put in the bag? Comparing and contrasting procedures for text clustering. Italian Journal of Applied Statistics/Statistica Applicata, 22(1), 77-94. 


\section{Tuzzi, A., \& Cortelazzo, M.A. (2018a). What is Elena Ferrante? A compara- tive analysis of a secretive bestselling Italian writer. Digital Scholarship in the Humanities, 33(3), 685-702. doi: doi.org/10.1093/1lc/fqx066. \\ Tuzzi, A., \& Cortelazzo, M.A. (Eds.). (2018b). Drawing Elena Ferrante's Pro- file. Workshop Proceedings, Padova, 7 September 2017. Padova: Pa- dova University Press. \\ Viprey, J.-M., \& Ledoux C.-N. (2006). About Labbé's intertextual distance. Journal of Quantitative Linguistics, 13(2-3), 265-283. doi: https://doi. org/10.1080/09296170600855444.}

Riassunto: Sulla base di un corpus di 150 romanzi di 40 autori, abbiamo condotto un'indagine stilometrica, per valutare quali autori contemporanei risultassero più vicini a Elena Ferrante, pseudonimo con cui sono stati firmati otto romanzi, tra cui l'Amica geniale (Tuzzi \& Cortelazzo 2018a e 2018b). L'indagine ha dimostrato che lo stile di scrittura di Elena Ferrante è notevolmente diverso da quello degli altri principali romanzieri italiani contemporanei, con la sola eccezione di Domenico Starnone. Studi successivi (Cortelazzo, Mikros \& Tuzzi 2018 e in preparazione) hanno mostrato che, invece, le opere non letterarie firmate da Elena Ferrante possono essere attribuite ad autori diversi, tra i quali Anita Raja, di nuovo Starnone e autore collettivo costituito dallo staff della casa editrice $\mathrm{E} / \mathrm{O}$.

Questo studio integra i risultati delle ricerche precedenti, aggiornando la collocazione di Elena Ferrante nel panorama contemporaneo, in seguito all'uscita del suo ultimo romanzo (La vita bugiarda degli adulti) e affinando lo studio delle similarità con Domenico Starnone, utilizzando un corpus più ampio di opere di questo autore. Risultano rafforzati i risultati delle ricerche precedenti.

Parole chiave: Elena Ferrante, letteratura italiana contemporanea, attribuzione d'autore, misure di similarità, classificazione dei testi 